\title{
La langue en question sous la coupole
}

\section{Christoph Hänggeli}

Avocat, directeur de l'Institut suisse pour la formation médicale postgraduée et continue (ISFM)

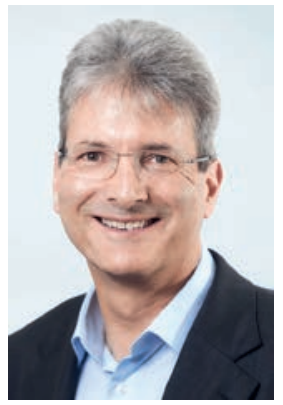

Chaque année, pas moins de 3000 médecins font reconnaître leurs diplômes étrangers par la Commission des professions médicales (MEBEKO). En Suisse, aujourd'hui, un médecin sur trois possède un diplôme étranger. Le nombre de médecins originaires de pays où l'allemand, le français et l'italien ne sont pas parlés a fortement augmenté au cours des dix dernières années, tout comme les problèmes de communication. Le manque de compréhension entre le médecin et le patient, ou au sein d'une équipe, augmente le risque de situations potentiellement dangereuses. C'est la raison pour laquelle la FMH et l'ISFM sont intervenus avec énergie dans le cadre de la loi sur les professions médicales (LPMéd) en faveur d'une réglementation efficace visant à garantir les compétences linguistiques, avec toutefois un succès mitigé. Si la LPMéd mentionne désormais «des connaissances linguistiques nécessaires à l'exercice de la profession", personne ne s'attendait à ce que le Conseil fédéral n'accepte que les trois options suivantes dans le droit d'exécution pour prouver les compétences linguistiques: un diplôme de langue reconnu au niveau international, qui ne doit pas dater de plus de six ans (au moins B2); un diplôme universitaire ou un titre postgrade obtenu dans la langue correspondante; ou une expérience professionnelle en tant que médecin de trois ans dans la langue correspondante.

Les obstacles bureaucratiques affectent potentiellement tous les médecins suisses souhaitant exercer dans une autre région linguistique.

Paradoxalement, ce sont précisément les médecins suisses souhaitant exercer dans une autre région linguistique qui font les frais d'une telle réglementation dénuée de bon sens. Même s'ils ont obtenu leur maturité, ils doivent présenter un diplôme de langue supplémentaire dans la langue concernée. A titre d'exemple, le Dr Ulrich Nägeli décrit dans ce numéro son combat kafkaïen avec les autorités qui ne veulent inscrire ses connaissances linguistiques dans le registre que sur présentation d'un diplôme de langue reconnu au niveau international alors que ses connaissances de l'italien sont largement supérieures au B2 requis (cf. page 860). Le cas du Dr Nägeli n'est pas un cas isolé. Les obstacles bureaucratiques saugrenus affectent potentiellement tous les médecins qui souhaitent exercer dans une autre région linguistique. La solution serait pourtant simple. Le Conseil fédéral n'aurait qu'à com- pléter l'ordonnance par une phrase indiquant que le certificat de maturité suisse suffit à prouver les compétences linguistiques. Mais il a déjà rejeté une motion dans ce sens déposée le 25 septembre 2018 par la conseillère nationale Regine Sauter, la jugeant «non opportune». Il n'a donc pas respecté la volonté du Parlement qui, lors des délibérations du projet de loi, a considéré que la maturité suisse était une preuve suffisante. Les examens de langue de la maturité correspondent au niveau B2. Le plan d'études des écoles secondaires du canton de Berne prévoit même le niveau B2/C1 pour le français.

Les Tessinois sont particulièrement concernés parce qu'ils étudient généralement en dehors de leur canton et obtiennent donc un diplôme de formation en allemand ou en français. Comble de l'absurde, s'ils veulent exercer au Tessin, ils sont obligés de payer pour faire inscrire leur propre langue maternelle dans le registre! Afin de balayer cette discrimination, le conseiller national Marco Chiesa a déposé le 6 mars 2019 une motion supplémentaire, signée par tous les conseillers nationaux tessinois, exigeant que la reconnaissance des compétences linguistiques soit simplifiée et offerte gratuitement.

Ces deux motions n'ont pas encore été inscrites à l'ordre du jour du Conseil national et pourraient connaître le même sort que de nombreuses autres motions non traitées: disparaître automatiquement après deux ans.

L'espoir semble venir d'ailleurs: le 3 juin 2019, le Conseil des Etats a pris une décision mémorable dans le cadre de la réglementation des admissions en discussion depuis des années; il ne se satisfait pas des preuves linguistiques telles que mentionnées dans la LPMéd mais exige explicitement un examen de langue passé en Suisse (C1). Et ce n'est pas tout: «Les médecins titulaires d'une maturité suisse [...] sont exemptés de cette obligation.» Si le Conseil national approuve le projet du Conseil des Etats, il serait plus qu'opportun que le Conseil fédéral adapte l'ordonnance en conséquence. Le dédale administratif marquerait alors enfin le pas.

\section{Références}

1 Hänggeli C. Les connaissances linguistiques aux limites de l'absurde. En 1877, la politique était-elle plus clairvoyante qu'en 2018 ? Bull Med Suisses. 2018;99(16):497.

2 Hänggeli C, Kuhn H. La LPMéd révisée entre en vigueur le $1^{\text {er }}$ janvier 2018. Bull Med Suisses. 2017;98(51-52):1727-8. 